\title{
Recent advances in micro detectors for micro gas chromatography
}

\author{
Hemi Qu ${ }^{1,2,3}$ and Xuexin Duan ${ }^{1,3^{*}}$
}

\begin{abstract}
Micro gas chromatography ( $\mu \mathrm{GC}$ ) has been continuously gaining attention since the last century owing to multiple favorable characteristics, such as its small size, low power consumption and minimal production and maintenance costs. $\mu \mathrm{GC}$ has the potential to provide practical solutions to emerging analytical challenges in security, health, and environment. In this review, we summarize recent advances in micro detectors for $\mu \mathrm{GC}$, including the study of the miniaturization of conventional detectors and the development of novel detectors for $\mu \mathrm{GC}$ chromatography.
\end{abstract}

Keywords: micro detector, gas sensor, micro gas chromatography, micro-electro-mechanical system

\section{INTRODUCTION}

Gas chromatography (GC) is an important analytical technique for gas analysis in many fields, including pharmaceuticals [1], foods [2], the environment [3] and the petroleum industry [4]. With ever-increasing analytical demands for the vapor detection in the fields of security [5], health [6] and space exploration [7], micro gas chromatography ( $\mu \mathrm{GC})$ has continuously attracted attention from the academics and industry since Terry et al. [8] demonstrated the first silicon-based microcolumn integrated with a thermal conductivity detector (TCD) in the late 1970s. The reason for such attention is that $\mu \mathrm{GC}$ offers multiple favorable characteristics, such as a small footprint, low power consumption and minimal production and maintenance costs, which are beneficial for the real-time qualitative and quantitative analysis of complex gas mixtures.

The performance of conventional GC systems relies heavily on the detector. During the development of GC, intensive interest and efforts have been dedicated to the fabrication of high-performance detectors, producing a variety of devices ranging from electrical to optical and acoustic detectors. For $\mu \mathrm{GC}$, the detector needs to be small in size and match the small flow dimensions within the microfluidic channel. With the advances in microtechnology, several micro detectors for $\mu \mathrm{GC}$ have been successfully fabricated by miniaturizing conventional counterparts. Additionally, several novel micro detectors based on new principles have been prepared and reported in the literature, even demonstrating the potential of measuring eluates at the single-molecule level [9].

This review will not cover all aspects of $\mu \mathrm{GC}$; instead, we will focus our attention on micro detectors. For the research covering all the components of $\mu \mathrm{GCs}$, Mello et al. [10] reviewed the early development of chip-based gas chromatography systems from 1980 to 2000. Lewis et al. [11] reviewed the advances represented by MicroChemLab, developed by Sandia National Laboratories in 2006. Haghighi et al. [12] investigated the developments and achievements in the field of chip-based GC and the main components thereof from the beginning of the field until 2015. Recently, Azzouz et al. [13] briefly reviewed microelectromechanical systems (MEMS)-based gas chromatography systems. Ghosh et al. [14] reviewed the developments in microchip GC column technology and in interfacing GC microchips to injectors and detectors. In this review, some general aspects of detectors will be introduced first, and then the miniaturization of conventional detectors and the development of new detectors for $\mu \mathrm{GC}$ will be investigated.

\section{GENERAL ASPECTS OF DETECTORS}

Detectors for GC are characterized by several parameters, including noise, sensitivity, selectivity, limit of detection, and dynamic range. For clarity in this review, some key

\footnotetext{
${ }^{1}$ State Key Laboratory of Precision Measuring Technology \& Instruments, Tianjin University, Tianjin 300072, China

${ }^{2}$ Key Laboratory of Advanced Energy Materials Chemistry (Ministry of Education), Nankai University, Tianjin 300071, China

${ }^{3}$ College of Precision Instrument and Opto-electronics Engineering, Tianjin University, Tianjin 300072, China

${ }^{*}$ Corresponding author (email: xduan@tju.edu.cn)
} 
parameters will be briefly discussed in this section. For more details, please refer to the book [15].

\section{Sensitivity and selectivity}

Detectors for gas chromatography are concentrationsensitive or mass-sensitive. The sensitivity $(S)$ of a detector is defined as the change in the detector output signal produced in response to a change in the mass or concentration of the eluted solute. Sensitivity is useful in comparing detectors of the same type.

Detectors in chromatography can be universally responsive or selective to eluates. For example, thermal conductivity detectors (TCDs) are universal detectors since they respond to all compounds, including the carrier gas, and electron capture detectors (ECDs) are selective since they only respond to compounds with high electron affinity.

\section{Noise and limit of detection}

Fig. 1 shows three parameters: peak height $(h)$, peak width at half-height $\left(W_{\mathrm{h}}\right)$ and noise $(N)$. The noise is the average signal output of the baseline over a period of time. The limit of detection (LOD) can be obtained by measuring the noise level, the sensitivity, and the peak width at half-height for the component of interest in the chromatogram [15].

\section{Dynamic range}

The dynamic range is the ratio between the largest and smallest values that a detector can measure. Generally, the linear dynamic range for a detector is the most relevant parameter and defines the range of concentration or mass over which the sensitivity of the detector is nearly constant.

\section{MICRO FLAME IONIZATION DETECTOR ( $\mu$ FID)}

FIDs are the most popular detection technology in gas chromatography owing to their multiple advantages, such as high sensitivity, good dynamic range, low cost and low maintenance requirements. FIDs work by the combustion of organic compounds in a hydrogen flame to produce ions, which are proportional to the mass of organic species in the sample gas stream and are measured by a collector electrode. Since combustion requires hydrogen and an oxidant, an extra hydrogen tank and extra input oxygen are necessary for GC systems with FIDs. Several groups are continuously working on the miniaturization of FIDs to reduce gas consumption in portable gas chromatographs. For example, Kuipers et al. [16,17]

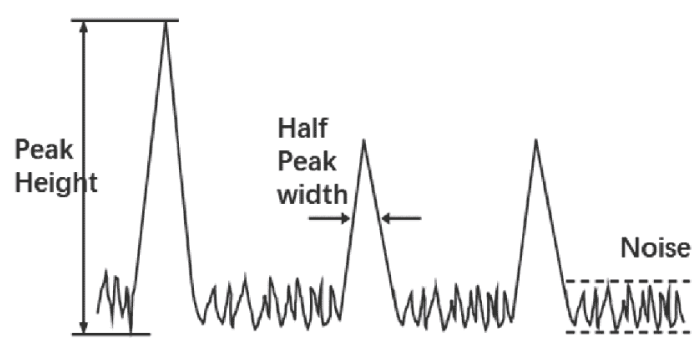

Figure 1 A chromatogram indicating peak height, peak width at half height, and noise.

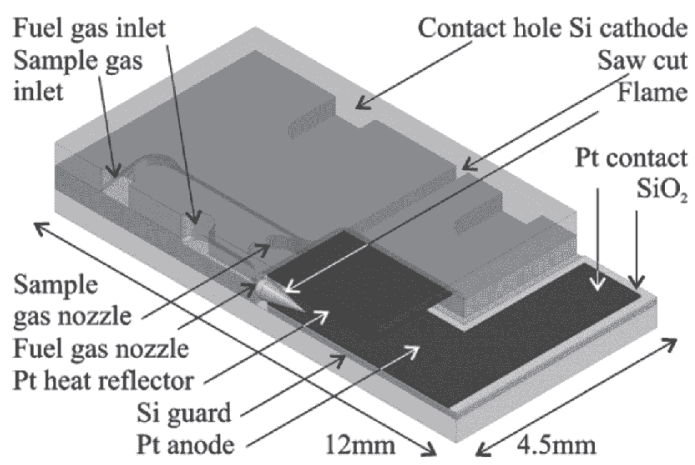

Sample gas inlet Fuel gas inlet Pt heat reflector

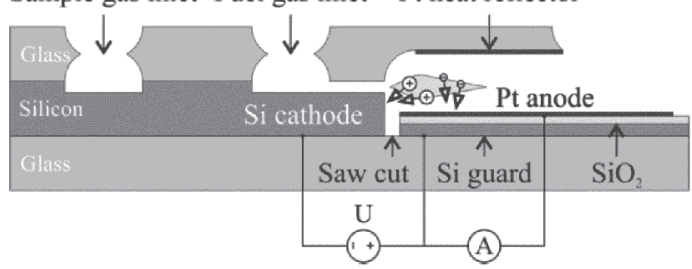

Figure $23 \mathrm{D}$ representation of half of a planar $\mu$ FID (top) and crosssectional view of a planar $\mu$ FID (bottom) $[16,17]$. Reproduced with permission from Ref. [16], Copyright 2011, Elsevier and Ref. [17], Copyright 2010, Elsevier.

reported the miniaturization of an FID by MEMS technology, as shown in Fig. 2. This micro detector is composed of cathode/anode electrodes and a heat reflector integrated within a microchannel. As seen in Fig. 2 (bottom), the fuel and sample gases are burned at the outlet of the silicon cathode, and the generated ions are collected on both the cathode and anode, therefore producing signals proportional to the mass of analytes in the sample flow. With this $\mu$ FID, the oxidant gas consumption is reduced considerably from $300 \mathrm{~mL} \mathrm{~min}^{-1}$ of air to $13 \mathrm{~mL} \mathrm{~min}^{-1}$ of pure oxygen without losing sensitivity. The consumption of hydrogen is $7.5 \mathrm{~mL} \mathrm{~min}{ }^{-1}$, different from the $30 \mathrm{~mL} \mathrm{~min}{ }^{-1}$ for conventional FID. In Kuiper's $\mu$ FID, a premixed flame with oxygen and hydrogen is used. Diffusion flames are 
generally known to be more controllable and stable than premixed flames. Shannon et al. [18] proposed a $\mu$ FID based on a diffusion flame that features a folded flame structure. This $\mu$ FID shows high ionization efficiency $\left(1.959 \times 10.2 \mathrm{C} \mathrm{mol}^{-1}\right.$ for methane) with low leakage of the analytes. However, the gas consumption is high and needs to be improved for portable applications. In this device, the flow rate of both air and hydrogen was reported to be $80 \mathrm{~mL} \mathrm{~min}^{-1}$. Currently, $\mu$ FIDs are inferior to full-size FIDs because the hydrogen flame loses the potential to generate ions at the reduced size. In contrast to FIDs, which are mass-sensitive, TCDs are concentration-sensitive and more suited for miniaturization.

\section{MICRO TCD ( $\mu$ TCD)}

TCDs consist of two parallel channels, containing gas and heating coils. Changes in the thermal conductivity of the eluate channel are measured and compared with the conductivity of the reference channel. To meet the requirements for $\mu \mathrm{GC}$, TCDs are continuously being optimized in terms of size, structure and fabrication process. The results of conventional TCDs generally need to be calculated with the flow rate of each gas in the gas stream. Recently, a dual-MEMS TCD was reported, where a reference chamber was used for flow compensation, thus avoiding the time-consuming recalibration process [19]. Cruz et al. [20] fabricated a $\mu$ TCD to analyze the effluent from a $\mu \mathrm{GC}$ column. This detector was composed of two identical microflow cells, namely, an analytical flow cell and a reference flow cell, to form a Wheatstone bridge circuit. The difference in heat between two flows could be measured with this Wheatstone bridge circuit by examining the change in resistance and was proportional to the concentration of analytes in the analytical flow. The side and top views of one microflow cell design are shown schematically in Fig. 3a, b. The heating resistor for the $\mu \mathrm{TCD}$ rests on a suspended silicon nitride membrane in pyramidal or trapezoidal flow cells (Fig. 3c, d). The suspended structure is beneficial in reducing power consumption. This $\mu \mathrm{TCD}$ features a two-thermistor thermal conductivity cell. Sun et al. [21] fabricated a polydimethylsiloxane (PDMS)-covered four-thermistor thermal conductivity cell, demonstrating an improved detector response compared with that obtained in a tworesistor system. However, this PDMS-coated device was difficult to realize in batch fabrication and therefore presented challenges in achieving device consistency. Later, this group reported a silicon-glass $\mu \mathrm{TCD}$ with fourthermistor thermal conductivity cells by replacing PDMS with glass in a standard MEMS fabrication process [22].
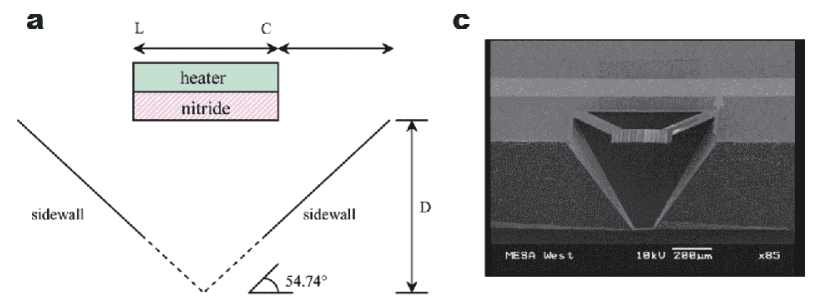

b
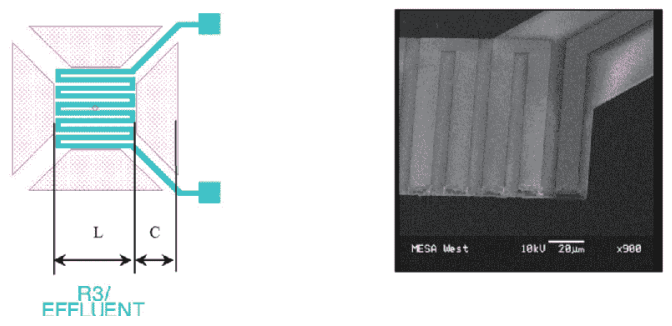

Figure 3 Cross-sectional and top-down views of a $\mu \mathrm{TCD}$ with a heater resting on a silicon nitride membrane [20]. Reproduced with permission from Ref. [20], Copyright 2007, Elsevier.

Currently, MEMS $\mu \mathrm{TCD}$, which is capable of measuring vapors down to $1 \mathrm{ppm}$, is commercially available and employed in several portable GC systems [23].

The monolithic integration of a high-sensitivity detector with a separation column eliminates several interconnections that can cause crucial errors and loss of fidelity during detection and analysis. Kaanta et al. [24] reported a monolithic GC separation column and $\mu \mathrm{TCD}$. This integrated device is able to separate nitrogen, methane and carbon dioxide within $30 \mathrm{~s}$. To reduce the number of fluidic ports, Narayanan et al. [25] embedded the reference resistor of the $\mu \mathrm{TCD}$ close to the inlet of the separation column in the integrated device. The resistors in this $\mu \mathrm{TCD}$ are anchored to the substrate and characterized by a poor LOD. Later, this group reported a suspended coiled metal resistor formed by sacrificial etching of silicon, demonstrating reduced power consumption, a quicker time response, and improved detection [26].

\section{MICRO PHOTOIONIZATION DETECTOR ( $\mu$ PID)}

PIDs are efficient and inexpensive detectors in conventional GC and can be used in portable gas analytical devices [27]. A typical photoionization detector consists of a UV source and an ionization chamber. UV radiation leads to analyte ionization, and the ionized species are measured by a collector electrode. Recently, Fan et al. [28] developed a micro photoionization detector by assembly with a krypton UV lamp; the built-in lamp drives the 


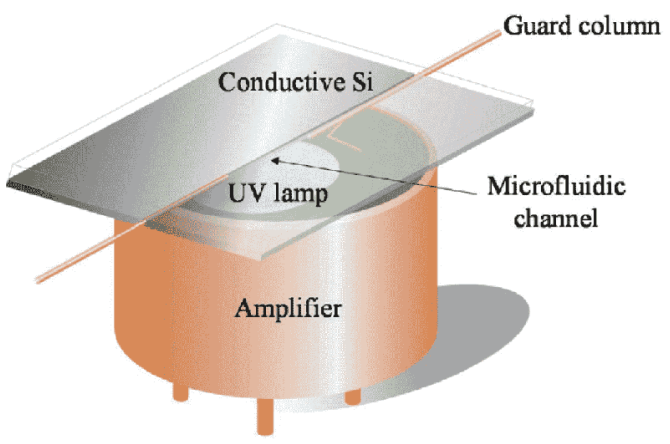

Figure 4 Schematic of a homemade $\mu$ PID with a built-in lamp drive circuit and amplifier from a commercial PID. Reproduced with permission from Ref. [28], Copyright 2016, the Royal Society of Chemistry.

circuit and the amplifier in a commercial PID as well as a homemade flow-through ionization chamber. The $\mu \mathrm{PID}$ shown in Fig. 4 employs a 2-cm long straight microfluidic channel in silicon wafers. The bottom and top of the microfluidic channel are covered with a krypton UV lamp and a glass slide. In this configuration, analytes are first ionized in the microchannel by UV radiation. Then, the conductive silicon serves as a signal collection electrode and measures the ionized species to produce signals. With this detector, this group demonstrated the first comprehensive portable automated 2-D GC instrument [29].

\section{PIEZOELECTRIC RESONATOR MICROSENSORS}

Piezoelectric resonator microsensors, with their excellent mass resolution, are an upcoming sensing technique for applications in chemical detection [30]. Piezoelectric resonator microsensors, including cantilevers, surface acoustic waves and bulk acoustic waves, are operated in the frequency range from $10^{3}$ to $10^{10} \mathrm{~Hz}$. In a gaseous environment, analyte molecules adsorb on the resonator surface, and the added mass induces changes in the resonating frequency, which are recorded as sensor signal output. For surface acoustic wave (SAW) and film bulk acoustic wave resonator (FBAR) sensors, the relation between frequency shift and mass change is specified by Sauerbrey equation:

$\Delta f=-\frac{2 f_{0}^{2}}{A \sqrt{\rho_{\mathrm{q}} \mu_{\mathrm{q}}}} \Delta m$

$S_{\mathrm{m}}=-\lim _{\Delta m \rightarrow 0} \frac{\Delta f / f_{0}}{\Delta m / A}$,

where $\Delta f$ and $f_{0}$ are the frequency shift and the resonant frequency, respectively; $\mu_{\mathrm{q}}$ and $\rho_{\mathrm{q}}$ are the elastic modulus and the density of piezoelectric material, respectively; $A$ is the area of the plate; $\Delta m$ is the deposited mass; and $S_{\mathrm{m}}$ indicates mass sensitivity. As sensor sensitivity is related to the mass per unit area, a reduced size in piezoelectric resonators will not result in a loss of sensitivity. Therefore, such components are highly suitable for miniaturization and lab-on-a-chip applications. Additionally, piezoelectric resonators have several other advantages, including high sensitivity towards surface perturbations, good linearity with low hysteresis, small size, and a relatively low cost. Several groups have reported piezoelectric resonator microsensors as sensitive detectors for $\mu \mathrm{GC}$.

\section{SAW sensors}

Sandia National Laboratories studied SAWs for $\mu$ TCD in 1996 [11]. The device used in their study is a delay line structure with a sensitive layer deposited between the two IDT ports. In their prototype hand-held gas chromatography instrument, named the MicroChemLab system, a four-element array of SAW detectors is employed to analyze eluents from the column. In this array, one device is blank and used as a control, and the other three devices are coated with polymer. Typical coating polymers include BSP3, PECH, ethyl cellulose and DKAP. The main advantage of polymer-coated SAW microsensor arrays as GC detectors is that the response patterns from the array can be used to identify eluting compounds in conjunction with mathematical methods. Recently, SAW microsensors were coupled with fast chromatography, resulting in a commercially available portable system named Z-Nose [31]. This system has several potential advantages in identifying contraband, hazardous vapors, and explosive and flammable materials in the gas phase.

\section{FBAR}

FBARs are typical piezoelectrical MEMS devices with a thin piezoelectrical layer sandwiched between top and bottom electrodes (Fig. 5). Similar to SAW gas sensors, coating sorptive materials on FBARs can improve sensitivity and selectivity, and sensor arrays using FBARs can quantitatively and qualitatively identify GC eluates [32-35]. In contrast to SAW, FBAR is much smaller in size with a high resonating frequency $(1-10 \mathrm{GHz})$. While the small size indicates that FBAR is well suited for integration in a microsystem, the high resonating frequency could endow FBAR with higher sensitivity when used as a GC detector. We first reported the facile hyphenation of FBAR with a commercial separation column in a prototype chromatographic instrument, demonstrating the potential advantages of FBAR as a 

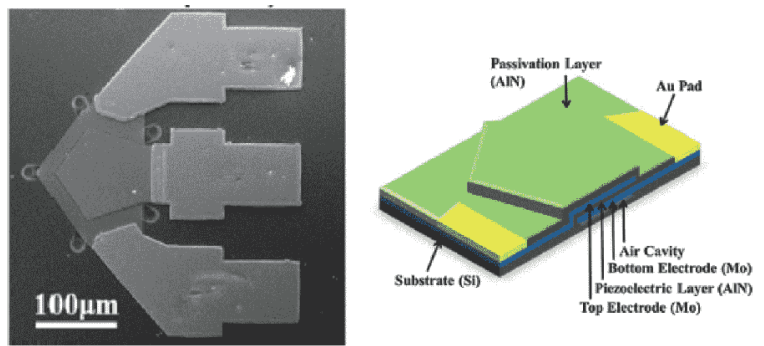

Figure 5 SEM image of a film bulk acoustic resonator (left) and structure schematic (right) [36,37]. Reproduced with permission from Ref. [36], Copyright 2018, Elsevier; Reproduced with permission from Ref. [37], Copyright 2016, American Chemical Society.

miniatured sensitive detector in $\mu \mathrm{GC}$ [36]. Coating the detector with nanomaterials (metal organic frameworks $(\mathrm{MOFs})$ ) significantly enhanced the detector response (20 times). Later, we showed that different polymer-coated FBAR sensor arrays could provide quantitative detection and identify overlapping dual mixtures through the PCA method in GC [37].

\section{Nanoelectromechanical system (NEMS) resonator}

Piezoelectric resonators such as SAW and FBAR are generally classified as MEMS. NEMS are the next logical step for further miniaturization. Fig. 6 shows typical NEMS cantilevers used for vapor detection. This resonant NEMS is extremely sensitive because of its unique attributes, such as its very small mass and high quality factor. Attainment of zeptogram resolution was demonstrated in 2006 by Roukes et al. [38] who later coupled a NEMS resonator with fast chromatography, demonstrating the detection of sub-parts per billion (ppb) concentrations of phosphonate analytes in $5 \mathrm{~s}$ [39]. In all mass-sensitive resonators, including SAW and FBAR, the chemical specificity of the sensor can be tuned by varying the type of polymer deposited on the NEMS. In the referenced study, a DKAP-coated device selectively responded to organophosphate eluates and thus was good for the detection of chemical warfare agents (CWAs). For the use of NEMS resonators as detectors in $\mu \mathrm{GC}$, the interaction cross-section with gas analytes can rapidly decrease as the active mechanical element decreases. To overcome this challenge, Roukes et al. [40] developed arrays of NEMS by large-scale integration, comprising thousands of individual NEMS resonators with densities of up to 6 million devices per square centimeter. The NEMS sensor arrays increase the interaction cross-section substantially and can measure vapor analytes collectively, demonstrating successful detection of organophosphates

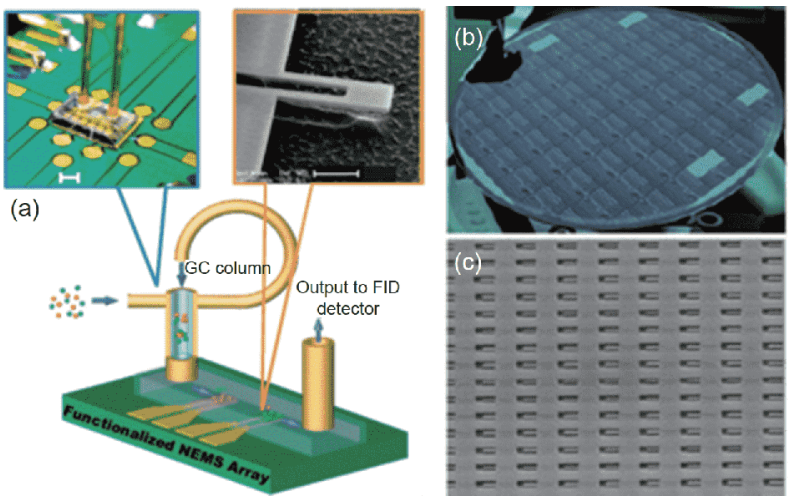

Figure 6 NEMS resonator. (a) Schematic view of an array of NEMS resonators encapsulated in a micromachined flow channel connected with a GC column. Right inset: Scanning electron micrograph of a cantilever NEMS resonator. (b) Photograph of a full $200 \mathrm{~mm}$ wafer with patterned NEMS arrays. (c) Scanning electron micrograph of a section of a cantilever array [39,40]. Reproduced with permission from Ref. [39], Copyright 2010, American Chemical Society; Reproduced with permission from Ref. [40], Copyright 2012, American Chemical Society.

at part-per-billion concentrations in $2 \mathrm{~s}$. Integration is very important for further miniaturization if a sensor will be used in $\mu \mathrm{GC}$. Ernst et al. [41] studied the integration of read-out circuits with NEMS resonators on the same chip, eliminating the need for interconnections in NEMS resonator arrays.

\section{MICRO PLASMA IONIZATION DETECTOR}

The micro plasma detector consists of a pair of discharge electrodes initiating the ionization of helium in the discharging region. The bypassing analytes are fragmented by the helium plasma, and the resulting ions are collected in the collector electrode to produce signals that are proportional to the mass of the analyte in the carrier gas (Fig. 7a). Narayanan et al. [42] reported a small $(20 \mathrm{~mm} \times 10 \mathrm{~mm})$ micro plasma device for the detection of gases in $\mu \mathrm{GC}$ in 2014. This device consists of discharge electrodes, bias electrodes and collector electrodes on a plate, as shown in Fig. 7a. After optimizing the design and operation parameters, the LOD was demonstrated to be $10 \mathrm{pg}$ for octane in air when applying a discharge power of $1.4 \mathrm{~mW}$. This performance is comparable with that of conventional FID detectors [43]. In addition, monolithic integration of this micro plasma detector with a separation column has been achieved through a twomask fabrication process [44]. The integrated chip is $1.5 \mathrm{~cm} \times 3 \mathrm{~cm}$ in size and alleviates the need for transfer lines between the column and the detector, which is beneficial in reducing the cost and improving the performance of GC. It should be noted that the 

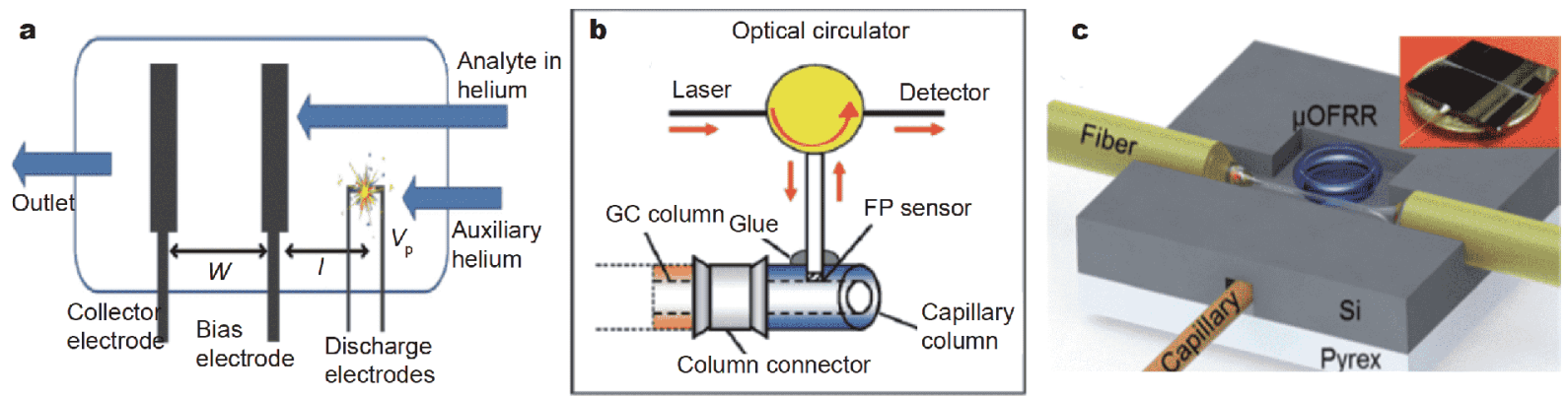

Figure 7 (a) Schematic setup to test the $\mu$ He-DPID [43]. (b) Schematic structure of the FP sensor module assembled by inserting an FP sensor probe into a hole drilled in a fused-silica capillary [46]. (c) Diagram of the $\mu$ OFRR with attached optical fiber and capillary connection (inset shows a photograph of the fully packaged device) [49,50]. Reproduced with permission from Ref. [43], Copyright 2015, Elsevier; Reproduced with permission from Ref. [46], Copyright 2010, American Chemical Society; Reproduced with permission from Ref. [49], Copyright 2014, the Royal Society of Chemistry; Reproduced with permission from Ref. [50], Copyright 2015, the Royal Society of Chemistry.

aforementioned detector utilizes direct current (DC) discharge to initiate helium plasma. The major drawback of using DC discharge is the limited lifetime of the detector because of the sputtering effect. A moderate decline in the baseline was observed in $24 \mathrm{~h}$ of operation [44]. To alleviate the sputtering effect, Fan et al. [45] developed a micro helium dielectric barrier PID where dielectric materials are placed on the electrode surface facing the discharge chamber as a protection layer. This detector is small in the area of only $15 \mathrm{~mm} \times 10 \mathrm{~mm}$ and the weight of only $0.25 \mathrm{~g}$. The LOD was demonstrated to be a few picograms. The device worked well during a 3week-long operation.

\section{FABRY-PÉROT (FP) CAVITY SENSOR}

In GC, tandem columns are beneficial for improving the separation capability and selectivity. However, the separated eluates from the first column can overlap again after passing through the second column. To overcome this problem, Fan et al. [46] employed an FP cavity sensor between tandem columns to achieve on-column detection. Thus, the separated eluates from the first column are detected before entering the second column. Fig. $7 \mathrm{~b}$ shows an FP cavity sensor composed of a thin layer of metal and sorptive polymer on the end face of a singlemode optical fiber. The working principle of FP cavity sensors is based on the frequency changes in response to analyte absorption. For example, the absorption of analytes in the polymer changes both the thickness and reflection index (RI) of the polymer film, resulting in a shift in the resonating wavelength in the interference pattern. The use of optical fibers causes a lack of control and variability in the deposition of gas-sensing polymer layers on the sensor. Later, this group developed an FP gas sensor fabricated on a silicon wafer and integrated a sensor array on one chip to improve the sensing capability for $\mu \mathrm{GC}$ applications [47]. The monolithic integration of sensor arrays with microcolumns was also achieved through standard MEMS fabrication process in a follow-up study [48].

\section{OPTICAL MICRO-RING RESONATOR}

Optical micro-ring resonator sensors consist of a waveguide near a micro-ring coating with a sorptive polymer (Fig. 7c). When a beam of light passes through a waveguide, a part of the light is resonated into the micro ring due to the evanescent wave field. The working principle of optical micro-ring resonators is similar to that of FP cavity sensors. The absorption of a gas analyte changes the RI and thickness of the polymer layer, in turn resulting in a shift in the resonating wavelength in the transmittance pattern. Conventional optical ring resonators are not well suited for microsystem integration because they are fragile and cumbersome. Zellers et al. [49] reported a fully functional microfabricated optofluidic ring resonator as a detector in $\mu \mathrm{GC}$. The detector was fabricated from silicon by batch-scalable micromachining techniques, integrating a PDMS-coated micro ring, a microfluidic channel, a capillary insertion port, and an optical-fiber alignment structure on a $4 \mathrm{~cm}^{2} \mathrm{Si}$ chip (Fig. 6c). A very low LOD for aromatics (0.5 ppm) was reported. This group later developed a nanoparticlecoated micro-optofluidic ring resonator as a detector for microscale GC [50]. Although the performance of sensors with nanoparticles is inferior in terms of detection limit, the use of arrays of monolayer protected metal nanoclusters (MPN)-coated sensors for compact $\mu \mathrm{GC}$ detectors shows some potential. 


\section{CHEMICAPACITIVE MICROSENSORS}

A "chemicapacitive" sensor is a capacitor with a selectively absorbing material, such as polymer, deposited as a dielectric on the electrodes [51,52]. The sensor measures the capacitance signal upon analyte absorption into the polymer, which changes the physical properties of the polymer layer, including the volume and dielectric constant. Patel et al. [51] developed a micromachined parallel-plate chemicapacitor, shown in Fig. 8 (top). Parallel-plate sensors consist of a layer of metal deposited on a substrate, followed by a layer of polymer and finally a second, porous layer of metal on top of the polymer. This type of sensor has demonstrated a detection limit in the low parts per million (ppm) range for most volatile organic compounds (VOCs) [51,53]. With this sensor, Seacoast Science Inc. [54] developed a portable chromatograph that could identify substances contained in volatile liquid or gaseous samples. Recently, Gianchandani et al. [82] fabricated a micro chromatograph for benzene, toluene and xylene (BTX) detection with a chemicapacitive sensor. This sensor is formed using closely spaced interdigitated electrodes covered with an OV-1 polymer layer (Fig. 8 (bottom)). With preconcentration, this sensor demonstrated a detection limit on the order of ppm in the analysis of 19 chemicals.

\section{GRAPHENE FIELD EFFECT TRANSISTOR (GrFET)}

Graphene-based transistors have developed rapidly and have been utilized for label-free chemical and biological sensors [55]. Fan et al. [56] reported a graphene nanoelectronic FET sensor as a detector in micro chromatography. Unlike DC-based FET sensing technology, their approach utilizes a GrFET in alternating current (AC) excitation, with surface-adsorbed molecules functioning as an oscillating gate (Fig. 9). This oscillating gate induces a conduction change as an output signal in the graphene channel. The use of AC excitation is beneficial in improving the sensing response. This GrFET sensor can simultaneously achieve rapid (down to $0.1 \mathrm{~s}$ ) and sensitive (down to $1 \mathrm{ppb}$ ) detection of a wide range of analytes in micro chromatography.

\section{CHEMIRESISTIVE GAS SENSORS}

Chemiresistive gas sensors consist of a sensing material that bridges the gap between two electrodes and measures resistance changes in the exposed vapor. Chemiresistive gas sensors are easily fabricated and integrated into micro chromatography. Chemiresistors with multiple sensitive materials, including metal oxide semiconductors, carbon

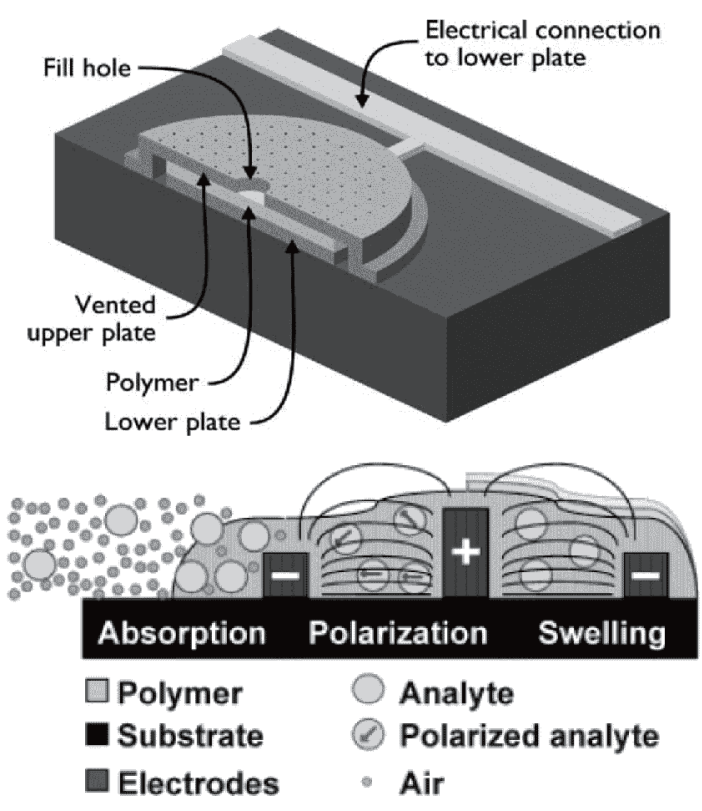

Figure 8 Schematic of a parallel-plate capacitive sensor (top) [51] and interdigitated capacitive sensor (bottom) [52]. Reproduced with permission from Ref. [51], Copyright 2003, Elsevier; Reproduced with permission from Ref. [52], Copyright 2004, American Chemical Society.

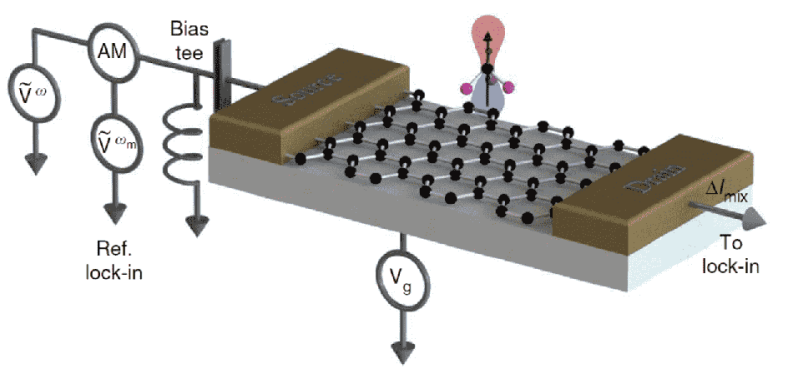

Figure 9 Schematic showing a graphene transistor configured as a highfrequency mixer for heterodyne vapor sensing and an illustration of a chloroform molecule on top of a graphene channel [56].

nanotubes, conductive polymers and monolayer-coated gold nanoparticle clusters, have been employed as single detectors or detector arrays in micro chromatography to address different vapor analysis challenges. While metal oxide semiconductor gas sensors generally require heating at high temperature, chemiresistors based on materials such as MPNs, carbon nanotubes (CNTs) and conductive polymers can operate at room temperature.

\section{Metal oxide semiconductor gas sensors (MOSs)}

MOSs are the most widely used sensors in gas detection and have been commercialized for several decades. MOSs offer several beneficial characteristics, such as low cost, 

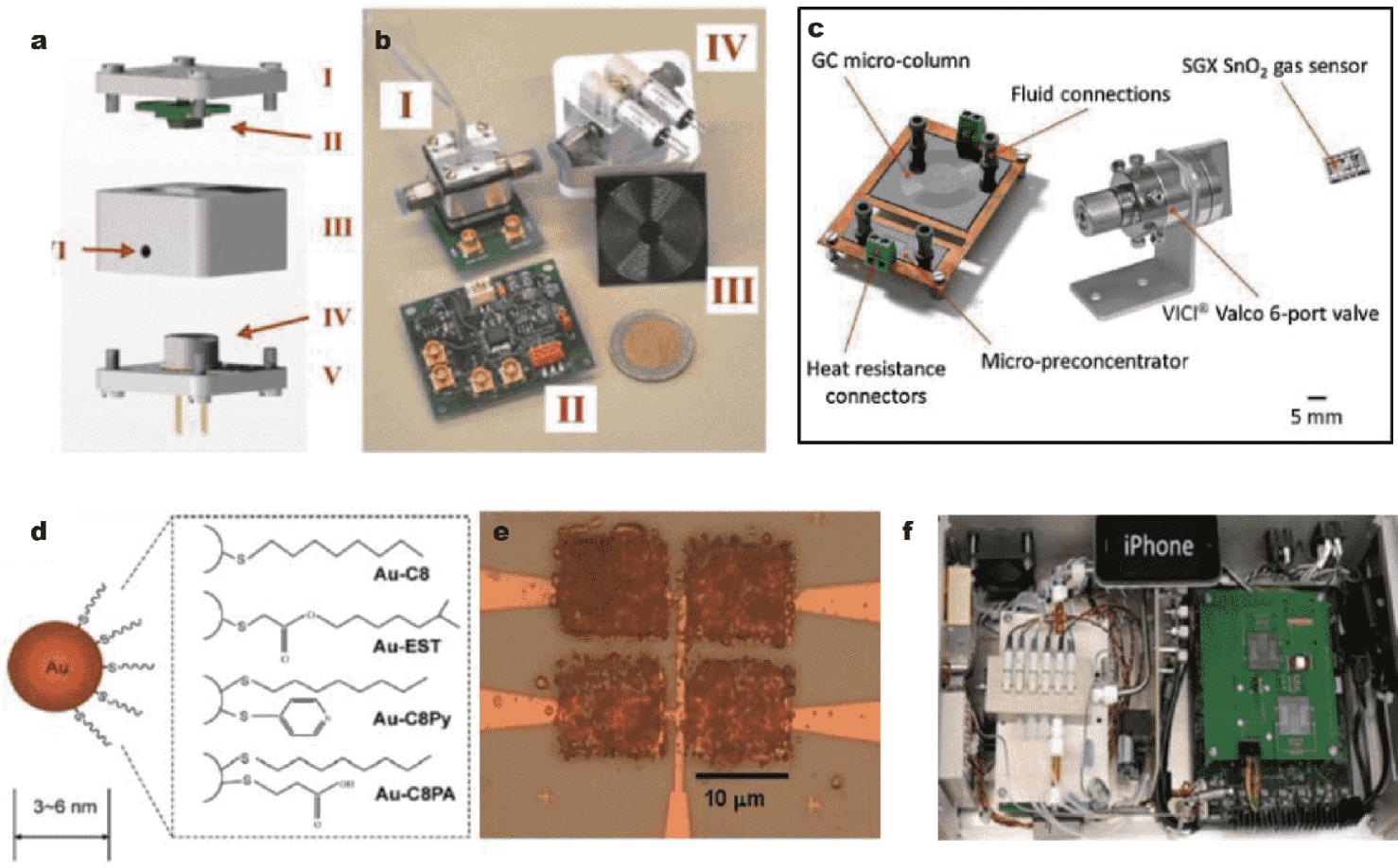

Figure 10 (a, b) Assembly of the entire $\mu$ GC-MOS sensor system and its components [61]. ((a) Detector chamber; I and V: top and bottom cover, III: main body with measurement chamber, IV: MOX gas sensor, II: SHT 15 temperature and humidity sensor, VI: sample inlet/outlet. (b) Used components; I: gas detector, II: detector electronics, III: GC-column chip, IV: integrated GC-column.) (c) Photographs of the e $\mu$ GC-MOS sensor with a commercial GC 6-port valve [64]. (d) Chemical structures of monolayer protected gold nanoclusters for the chemiresistor array [66]. (e) Optical micrograph of microdispenser-printed gold nanoclusters film covering all four CRs in a mono-MPN array [67]. (f) Prototype of $\mu$ GC-nanocluster sensor system [71]. Reproduced with permission from Ref. [61], Copyright 2013, Elsevier; Reproduced with permission from Ref. [64], Copyright 2018, Elsevier; Reproduced with permission from Ref. [66], Copyright 2012, Elsevier; Reproduced with permission from Ref. [67], Copyright 2011, American Chemical Society; Reproduced with permission from Ref. [71], Copyright 2012, American Chemical Society.

high sensitivity, and light weight [57]. Research in MOSs as detectors for $\mu \mathrm{GC}$ includes the following: 1) integrating MOSs with microcolumns, 2) improving measuring performance with new MOSs, and 3) addressing new analytical challenges by optimizing micro systems. Zampolli et al. [58] combined metal oxide gas sensors with a micromachined gas chromatographic column to enhance selectivity, demonstrating the successful detection of trace benzene, toluene, ethylbenzene and xylene (BTEX) (5 ppb) for indoor hazardous material monitoring. Dobrokhotov et al. [59] improved MOS performance by using gold-palladium (9:1 molar ratio) bimetal nanoparticle-decorated $\mathrm{SnO}_{2}$ thin films as sensitive materials. Ultralow concentrations of BTEX components at the sub-ppb level $(0.3 \mathrm{ppb})$ were identified and detected by this MOS sensor in GC analysis with a concentrator. The use of $\mathrm{SnO}-\mathrm{SnO}_{2}$ hybrid materials can detect benzene as low as 5 ppb without a concentrator [60]. In addition to BTEX analysis, $\mu \mathrm{GC}-\mathrm{MOS}$ sensor systems have been employed to analyze ethylene and alcohols. Sklorz et al.
[61] developed a miniaturized packed GC column and a $\mathrm{SnO}_{2}$ gas detector for the analysis of low-molecularweight hydrocarbons such as ethylene (Fig. 10a, b). Guan et al. [62] developed a portable GC with a highly sensitive and rapidly responsive semiconductor metal oxide detector based on an $\operatorname{In}_{2} \mathrm{O}_{3}$ nanoparticle film for the analysis of alcohols at concentrations as low as several ppb [63]. A promising area for the use of micro GC-MOS sensors is the onsite analysis of exhaled VOCs for disease diagnosis. Sanchez et al. [64] developed a $\mu$ GC with a single MOS as a detector for the detection of four biomarkers within 4 min with detection limits as low as $24,5,21$ and $112 \mathrm{ppb}$ for toluene, $o$-xylene, propanol and cyclohexane, respectively (Fig. 10c). Although this technique is promising, several limitations need to be addressed for practical applications in the future. These limitations include the following: 1) at a low $\mathrm{ppb}$ concentration, drift in the baseline of metal oxide gas sensors makes it difficult to measure the exact peak heights in the chromatogram;2) the required use of air as 
the carrier gas for MOS detectors can potentially cause stationary-phase degradation at elevated temperatures; and 3) humidity can deteriorate MOS performance over time when using air as the carrier gas.

\section{Chemiresistors based on metal nanoparticles}

In 2002, Zellers et al. [65] first introduced chemiresistors with MPNs as sensitive materials in GC. This group later demonstrated a high-performance portable GC with arrayed MPN chemiresistors. The sensor performance could be tuned by utilizing different monolayer coatings on the nanoparticles (Fig. 10d) [66]. To facilitate the integration of sensors in micro chromatography and generate sensor arrays in a limited space, this group explored an electron-beam-induced cross-linking method to create films with different MPNs on four sensors in a miniature array occupying an area of only $600 \mu^{2}$ (Fig. 10e) [67]. This reduction in sensor size significantly decreased the detector volume, achieving the ultralow dead volume required for $\mu \mathrm{GC}$ analyzers. To improve the portability of $\mu \mathrm{GC}$, Mason et al. [68] studied the monolithic implementation of CMOS readout circuits and MPN-coated CR arrays. Monolithically integrating such sensor arrays with CMOS instrumentation electronics on a single chip significantly enhances GC miniaturization and reduces detection noise. Several portable GC prototypes with MPN chemiresistors as detectors have been demonstrated in the literature for the detection of explosive markers [69], lung cancer VOC biomarkers [70], and indoor air pollutants (Fig. 10f) [71].

\section{Chemiresistors based on carbon nanotubes}

Because of their high surface-to-volume ratio and configuration as one-dimensional nanosystems, CNTbased devices are very promising as ultrasensitive chemical sensors [72]. A central problem with CNTbased sensors is that molecular adsorption is irreversible upon exposure to a wide range of analytes. Thus, the combination of a SWNT sensor and $\mu \mathrm{GC}$ column is not possible with such sensors. Strano et al. [73] improved sensor reversibility by functionalizing a SWNT network with polypyrrole. This newly developed sensor could detect a pulse of very dilute dimethyl methylphosphonate (DMMP) vapor (150 ppb) at the end of a $\mu \mathrm{GC}$ column. Alternatively, Masel et al. [74] improved reversibility by operating a device in current stimulated desorption (CSD) mode with uncoated CNTs as a sensitive layer. This uncoated CNT detector was comparable with FID in GC in the analysis of a mixture of nine gas analytes, including alcohols and aromatics.

\section{Chemiresistors based on conductive polymers}

Conducting polymers have many favorable characteristics for sensing, such as facile synthesis, good processability, varied functionality and, most importantly, roomtemperature operation [75,76]. Sadik et al. [77] fabricated a polypyrrole-based chemiresistor that was used as a detector for GC. Although the performance was inferior to that of PID detectors, their results indeed demonstrated the effectiveness of employing a polypyrrole-based chemiresistor as a GC detector. Alizadeh et al. [78] fabricated a chemiresistor based on dodecylsulfate-doped nanoporous polypyrrole by vapor deposition polymerization. Coupled with GC, this sensor could detect aromatic hydrocarbons at concentrations as low as $50 \mathrm{ppm}$. Pirsa et al. [79] later used polypyrrole with $\mathrm{ZnO}$ nanoparticles as sensitive materials in chemiresistors. As a detector in a portable GC, this sensor could detect trace aliphatic amines extracted from sewage and rainwater through a liquid-liquid microextraction process. Recently, we prepared a chemiresistor from conductive polymer nanowires fabricated by soft lithography [80]. This sensor showed fast response and high sensitivity, both of which are required for high-performance GC detectors.

\section{OUTLOOK}

As demonstrated in this review, significant progress has been made in recent decades, resulting in several portable GC instruments (Table 1). Many of those techniques have been made commercially available from instrument manufacturers, such as Inficon, Electronic Sensor Technology, Defiant-tech, and Agilent. In comparison with bulky and fragile benchtop GC, those instruments are much more suitable for addressing analytical challenges in the field. However, handheld instruments for on-site analysis are still elusive and not available in the market, even though the benchmark handheld MicroChemLab $\mu \mathrm{GC}$ from Sandia National Laboratories was demonstrated in the early 2000s. As a practical solution to new demanding tasks in breath gas analysis, space exploration, remote environmental monitoring and battlefield applications, micro chromatography needs to be further improved in size, performance and manufacturing. Enabled by rapidly evolving microtechnology, chip-scale GC could be the future in parallel with a paradigm shift from central-lab-based analysis to samplebased analysis. Accordingly, to meet the challenge ahead, detectors for micro chromatography need to be further developed in terms of miniaturization, sensitivity and 
Table 1 A list of micro-detectors with portable GC instrumentation

\begin{tabular}{|c|c|c|c|}
\hline Detector & Portable GC instrumentation & Comments on the detectors & Reference \\
\hline$\mu \mathrm{TCD}$ & $\begin{array}{l}\text { Carrier gas: helium; hydrogen; nitrogen; argon } \\
\text { Column: capillary column } \\
\text { Size and weight [81]: } 15 \mathrm{~cm} \times 45 \mathrm{~cm} \times 30 \mathrm{~cm}, \sim 3.5 \mathrm{~kg} \\
\text { Size and weight [23]: } 46.2 \mathrm{~cm} \times 19.6 \mathrm{~cm} \times 25.4 \mathrm{~cm} \text {, } \\
\sim 6.2 \mathrm{~kg} \\
\text { Power: } 100-240 \mathrm{~V}\end{array}$ & $\begin{array}{l}\text { Concentration sensitive and universally responsive; } \\
\text { good linearity; detection limit comparable with } \\
\text { conventional TCD; no need for auxiliary gas; } \\
\text { relatively easy to miniaturize; good candidate for } \\
\text { handheld GC }\end{array}$ & {$[23,81]$} \\
\hline$\mu \mathrm{FID}$ & $\begin{array}{l}\text { Carrier gas: electrolyzed for the generation of } \mathrm{H}_{2} \\
\text { and } \mathrm{O}_{2} \\
\text { Column: MEMS silicon column } \\
\text { Size and weight: } 24 \mathrm{~cm} \times 20 \mathrm{~cm} \times 10 \mathrm{~cm}, \sim 4 \mathrm{~kg} \\
\text { Power: Li-ion battery }\end{array}$ & $\begin{array}{l}\text { Mass sensitive with little/no response to inflam- } \\
\text { mable gases; good linearity; detection limit inferior } \\
\text { to that of conventional FID; needs auxiliary gas; } \\
\text { difficult to miniaturize; challenging for use in } \\
\text { handheld GC }\end{array}$ & {$[7]$} \\
\hline $\begin{array}{l}\text { MOS gas sensor } \\
\left(\mathrm{SnO}_{2}\right)\end{array}$ & $\begin{array}{l}\text { Carrier gas: synthetic air } \\
\text { Column: MEMS silicon column with concentrator } \\
\text { Size and weight: NA; Power: NA }\end{array}$ & $\begin{array}{l}\text { Concentration sensitive with tunable selectivity; } \\
\text { nonlinearity; detection limit down to sub-ppm; no } \\
\text { need for auxiliary gas; easy to miniaturize; mass } \\
\text { produced; good candidate for handheld GC }\end{array}$ & {$[59]$} \\
\hline Capacitive detector & $\begin{array}{l}\text { Carrier gas: purified air } \\
\text { Column: capillary column } \\
\text { Size and weight: } 10.8 \mathrm{~cm} \times 13.3 \mathrm{~cm} \times 19.1 \mathrm{~cm}, \sim 1.3 \mathrm{~kg} \\
\text { Power: } 100-240 \mathrm{~V} \text { AC power supply }\end{array}$ & $\begin{array}{l}\text { Concentration sensitive with tunable selectivity; } \\
\text { good linearity; detection limit down to ppm; no } \\
\text { need for auxiliary gas; easy to miniaturize; mass } \\
\text { produced; good candidate for handheld GC }\end{array}$ & {$[54,82]$} \\
\hline MPN chemiresistor & $\begin{array}{l}\text { Carrier gas: purified air } \\
\text { Column: MEMS silicon column with concentrator } \\
\text { Size and weight [71]: } 44 \mathrm{~cm} \times 25.5 \mathrm{~cm} \times 14.5 \mathrm{~cm}, \sim 4.5 \mathrm{~kg} \\
\text { Size and weight [70]: } 16 \mathrm{~cm} \times 11 \mathrm{~cm} \times 11 \mathrm{~cm}, \mathrm{NA} \\
\text { Power: main AC power supply }\end{array}$ & $\begin{array}{l}\text { Concentration sensitive with tunable selectivity; } \\
\text { good linearity; detection limit down to sub-ppm; no } \\
\text { need for auxiliary gas; easy to miniaturize; good } \\
\text { candidate for handheld GC }\end{array}$ & {$[70,71]$} \\
\hline SAW & $\begin{array}{l}\text { Carrier gas: helium } \\
\text { Column: capillary column } \\
\text { Size and weight: } 31.8 \mathrm{~cm} \times 26.4 \mathrm{~cm} \times 14.5 \mathrm{~cm}, \sim 8.5 \mathrm{~kg} \\
\text { Power: battery }\end{array}$ & $\begin{array}{l}\text { Concentration sensitive with tunable selectivity; } \\
\text { good linearity; detection limit down to ppm; no } \\
\text { need for auxiliary gas; easy to miniaturize; mass } \\
\text { produced; good candidate for handheld GC }\end{array}$ & {$[31]$} \\
\hline$\mu \mathrm{PID}$ & $\begin{array}{l}\text { Carrier gas: scrubbed ambient air } \\
\text { Column: NA } \\
\text { Size and weight: } 28 \mathrm{~cm} \times 20 \mathrm{~cm} \times 30 \mathrm{~cm}, \sim 2.2 \mathrm{~kg} \\
\text { Power: battery }\end{array}$ & $\begin{array}{l}\text { Mass sensitive; applicable in aromatic hydrocarbon } \\
\text { analysis; good linearity; detection limit comparable } \\
\text { with that of the bulk device; no need for auxiliary } \\
\text { gas; needs light source; difficult to miniaturize; } \\
\text { challenging to use in handheld GC }\end{array}$ & {$[27,83]$} \\
\hline $\begin{array}{l}\mu \text { Plasma ionization } \\
\text { detector }\end{array}$ & $\begin{array}{l}\text { Carrier gas: helium } \\
\text { Column: semipacked MEMS silicon column } \\
\text { Size and weight: NA } \\
\text { Power: NA }\end{array}$ & $\begin{array}{l}\text { Mass sensitive; good linearity; detection limit } \\
\text { comparable with that of the bulk device; needs } \\
\text { auxiliary gas; difficult to miniaturize; challenging to } \\
\text { use in handheld GC }\end{array}$ & {$[44]$} \\
\hline
\end{tabular}

mass production. In the near future, we anticipate that mass-produced, small-footprint and high-performance detectors can be installed on handheld devices that are independent of extra gas tanks, battery powered, and automated.

\section{Received 09 November 2018; accepted 26 December 2018; published online 14 February 2019}

1 Citti C, Braghiroli D, Vandelli MA, et al. Pharmaceutical and biomedical analysis of cannabinoids: A critical review. J Pharmaceutical BioMed Anal, 2018, 147: 565-579

2 Petrarca MH, Godoy HT. Gas chromatography-mass spectrometry determination of polycyclic aromatic hydrocarbons in baby food using QuEChERS combined with low-density solvent dispersive liquid-liquid microextraction. Food Chem, 2018, 257: 44-52

3 Ohira SI, Toda K. Micro gas analyzers for environmental and medical applications. Anal Chim Acta, 2008, 619: 143-156
4 Oetjen K, Thomas L. Volatile and semi-volatile organic compound patterns in flowback waters from fracturing sites within the Marcellus Shale. Environ Earth Sci, 2016, 75: 1043

5 Kanamori-Kataoka M, Seto Y. Measurement of breakthrough volumes of volatile chemical warfare agents on a poly(2,6-diphenylphenylene oxide)-based adsorbent and application to thermal desorption-gas chromatography/mass spectrometric analysis. J Chromatography A, 2015, 1410: 19-27

6 Callol-Sanchez L, Munoz-Lucas MA, Gomez-Martin O, et al. Observation of nonanoic acid and aldehydes in exhaled breath of patients with lung cancer. J Breath Res, 2017, 11: 026004

7 Bae B, Kim J, Yeom J, et al. In Development of a portable gas analyzer using a micro-gas chromatograph/flame ionization detector (micro-GC/FID) for NASA'S environmental missions, $42^{\text {nd }}$ International Conference on Environmental Systems 2012

8 Terry SC, Jerman JH, Angell JB. A gas chromatographic air analyzer fabricated on a silicon wafer. IEEE Trans Electron Devices, 1979, 26: 1880-1886

9 Sage E, Brenac A, Alava T, et al. Neutral particle mass spectro- 
metry with nanomechanical systems. Nat Commun, 2015, 6: 6482

10 de Mello A. On-chip chromatography: The last twenty years. Lab Chip, 2002, 2: 48N-54N

11 Lewis KL, Lianyong Su KL, Hawkridge FM, et al. Immobilization of cytochrome $c$ oxidase into electrode-supported lipid bilayer membranes for in vitro cytochrome c sensing. IEEE Sensor J, 2006, 6: $420-427$

12 Haghighi F, Talebpour Z, Sanati-Nezhad A. Through the years with on-a-chip gas chromatography: a review. Lab Chip, 2015, 15: 2559-2575

13 Azzouz I, Marty F, Bourouina T. In recent advances in micro-gas chromatography-the opportunities and the challenges, 2017 Symposium on Design, Test, Integration and Packaging of MEMS/ MOEMS (DTIP), 29 May-1 June 2017, 2017, pp 1-5

14 Ghosh A, Vilorio CR, Hawkins AR, et al. Microchip gas chromatography columns, interfacing and performance. Talanta, 2018, 188: 463-492

15 Robert LG, Eugene FB, Modern Practice of gas chromatography. York: John Wiley \& Sons, 2004, 227-338

16 Kuipers W, Müller J. Characterization of a microelectromechanical systems-based counter-current flame ionization detector. J Chromatography A, 2011, 1218: 1891-1898

17 Kuipers W, Müller J. Sensitivity of a planar micro-flame ionization detector. Talanta, 2010, 82: 1674-1679

18 Kim J, Bae B, Hammonds J, et al. Development of a micro-flame ionization detector using a diffusion flame. Sensor Actuat B-Chem, 2012, 168: 111-117

19 de Graaf G, Abarca Prouza A, Ghaderi M, et al. Micro thermal conductivity detector with flow compensation using a dual MEMS device. Sensor Actuat A-Phys, 2016, 249: 186-198

20 Cruz D, Chang J, Showalter S, et al. Microfabricated thermal conductivity detector for the micro-ChemLab ${ }^{\mathrm{TM}}$. Sensor Actuators B-Chem, 2007, 121: 414-422

21 Sun J, Cui D, Chen X, et al. Design, modeling, microfabrication and characterization of novel micro thermal conductivity detector. Sensor Actuat B-Chem, 2011, 160: 936-941

22 Sun JH, Cui DF, Chen X, et al. A micro gas chromatography column with a micro thermal conductivity detector for volatile organic compound analysis. Rev Sci Instruments, 2013, 84: 025001

23 Portable GC Agilent 490 (https://www.agilent.com/), Micro GC Fusion ${ }^{\star}$ Gas Analyzer (https://www.inficon.com/)

24 Kaanta BC, Chen H, Zhang X. A monolithically fabricated gas chromatography separation column with an integrated high sensitivity thermal conductivity detector. J Micromech Microeng, 2010, 20: 055016

25 Narayanan S, Alfeeli B, Agah M. A micro gas chromatography chip with an embedded non-cascaded thermal conductivity detector. Procedia Eng, 2010, 5: 29-32

26 Narayanan S, Alfeeli B, Agah M. Two-port static coated micro gas chromatography column with an embedded thermal conductivity detector. IEEE Sensor J, 2012, 12: 1893-1900

27 Jian RS, Huang YS, Lai SL, et al. Compact instrumentation of a $\mu$ GC for real time analysis of sub-ppb VOC mixtures. Microchem J, 2013, 108: 161-167

28 Lee J, Zhou M, Zhu H, et al. In situ calibration of micro-photoionization detectors in a multi-dimensional micro-gas chromatography system. Analyst, 2016, 141: 4100-4107

29 Lee J, Zhou M, Zhu H, et al. Fully automated portable comprehensive 2-dimensional gas chromatography device. Anal Chem, 2016, 88: 10266-10274
30 Pang $\mathrm{W}$, Zhao $\mathrm{H}$, Kim ES, et al. Piezoelectric microelectromechanical resonant sensors for chemical and biological detection. Lab Chip, 2012, 12: 29-44

31 Z-Nose http://www.estcal.com/ Available at 2018.9.4

32 Chang Y, Hui Z, Wang X, et al. Dual-mode gas sensor composed of a silicon nanoribbon field effect transistor and a bulk acoustic wave resonator: a case study in freons. Sensors, 2018, 18: 343

33 Liu W, Qu H, Hu J, et al. A highly sensitive humidity sensor based on ultrahigh-frequency microelectromechanical resonator coated with nano-assembled polyelectrolyte thin films. Micromachines, 2017, 8: 116

34 Chang Y, Tang N, Qu H, et al. Detection of volatile organic compounds by self-assembled monolayer coated sensor array with concentration-independent fingerprints. Sci Rep, 2016, 6: 23970

35 Lu Y, Chang Y, Tang N, et al. Detection of volatile organic compounds using microfabricated resonator array functionalized with supramolecular monolayers. ACS Appl Mater Interfaces, 2015, 7: 17893-17903

36 Wang YW, Ao CY, Hui ZP, et al. In film bulk acoustic resonator based gas sensor: A sensitive detector for gas chromatography, TRANSDUCERS $2017-19^{\text {th }}$ International Conference on SolidState Sensors, Actuators and Microsystems, 2017, pp 1471-1474

$37 \mathrm{Hu}$ J, Qu H, Chang Y, et al. Miniaturized polymer coated film bulk acoustic wave resonator sensor array for quantitative gas chromatographic analysis. Sensor Actuat B-Chem, 2018, 274: 419-426

38 Yang YT, Callegari C, Feng XL, et al. Zeptogram-scale nanomechanical mass sensing. Nano Lett, 2006, 6: 583-586

39 Li M, Myers EB, Tang HX, et al. Nanoelectromechanical resonator arrays for ultrafast, gas-phase chromatographic chemical analysis. Nano Lett, 2010, 10: 3899-3903

40 Bargatin I, Myers EB, Aldridge JS, et al. Large-scale integration of nanoelectromechanical systems for gas sensing applications. Nano Lett, 2012, 12: 1269-1274

41 Martin O, Gouttenoire V, Villard P, et al. Modeling and design of a fully integrated gas analyzer using a $\mu \mathrm{GC}$ and NEMS sensors. Sensor Actuat B-Chem, 2014, 194: 220-228

42 Narayanan S, Rice G, Agah M. A micro-discharge photoionization detector for micro-gas chromatography. Microchim Acta, 2014, 181: 493-499

43 Narayanan S, Rice G, Agah M. Characterization of a micro-helium discharge detector for gas chromatography. Sensor Actuat BChem, 2015, 206: 190-197

44 Akbar M, Shakeel H, Agah M. GC-on-chip: integrated column and photoionization detector. Lab Chip, 2015, 15: 1748-1758

45 Zhu H, Zhou M, Lee J, et al. Low-power miniaturized helium dielectric barrier discharge photoionization detectors for highly sensitive vapor detection. Anal Chem, 2016, 88: 8780-8786

46 Liu J, Sun Y, Howard DJ, et al. Fabry-Perot cavity sensors for multipoint on-column micro gas chromatography detection. Anal Chem, 2010, 82: 4370-4375

47 Reddy K, Guo Y, Liu J, et al. On-chip Fabry-Pérot interferometric sensors for micro-gas chromatography detection. Sensor Actuat BChem, 2011, 159: 60-65

48 Reddy K, Liu J, Oo MKK, et al. Integrated separation columns and Fabry-Pérot sensors for microgas chromatography systems. J Microelectromech Syst, 2013, 22: 1174-1179

49 Scholten K, Fan X, Zellers ET. A microfabricated optofluidic ring resonator for sensitive, high-speed detection of volatile organic compounds. Lab Chip, 2014, 14: 3873-3880

50 Scholten K, Collin WR, Fan X, et al. Nanoparticle-coated micro- 
optofluidic ring resonator as a detector for microscale gas chromatographic vapor analysis. Nanoscale, 2015, 7: 9282-9289

51 Patel SV, Mlsna TE, Fruhberger B, et al. Chemicapacitive microsensors for volatile organic compound detection. Sensor Actuat BChem, 2003, 96: 541-553

52 Kummer AM, Hierlemann A, Baltes H. Tuning sensitivity and selectivity of complementary metal oxide semiconductor-based capacitive chemical microsensors. Anal Chem, 2004, 76: 24702477

53 Mlsna TE, Cemalovic S, Warburton M, et al. Chemicapacitive microsensors for chemical warfare agent and toxic industrial chemical detection. Sensor Actuat B-Chem, 2006, 116: 192-201

54 Seacoast Science: http://www.seacoastscience.com/

55 Fu W, Jiang L, van Geest EP, et al. Sensing at the surface of graphene field-effect transistors. Adv Mater, 2017, 29: 1603610

56 Kulkarni GS, Reddy K, Zhong Z, et al. Graphene nanoelectronic heterodyne sensor for rapid and sensitive vapour detection. Nat Commun, 2014, 5: 4376

57 Sun Z, Liao T, Kou L. Strategies for designing metal oxide nanostructures. Sci China Mater, 2017, 60: 1-24

58 Zampolli S, Elmi I, Stürmann J, et al. Selectivity enhancement of metal oxide gas sensors using a micromachined gas chromatographic column. Sensor Actuat B-Chem, 2005, 105: 400-406

59 Larin A, Womble PC, Dobrokhotov V. Novel highly-integrated mems based solid state detectors for analytical gas chromatography. Sensor Actuat B-Chem, 2018, 256: 1057-1068

60 Sun J, Geng Z, Xue N, et al. A mini-system integrated with metaloxide-semiconductor sensor and micro-packed gas chromatographic column. Micromachines, 2018, 9: 408

61 Sklorz A, Janßen S, Lang W. Application of a miniaturised packed gas chromatography column and a $\mathrm{SnO}_{2}$ gas detector for analysis of low molecular weight hydrocarbons with focus on ethylene detection. Sensor Actuat B-Chem, 2013, 180: 43-49

62 Meng $\mathrm{H}$, Yang W, Yan X, et al. A highly sensitive and fast responsive semiconductor metal oxide detector based on $\operatorname{In}_{2} \mathrm{O}_{3}$ nanoparticle film for portable gas chromatograph. Sensor Actuat BChem, 2015, 216: 511-517

63 Khalid T, White P, De Lacy Costello B, et al. A pilot study combining a GC-sensor device with a statistical model for the identification of bladder cancer from urine headspace. PLoS ONE, 2013, 8: e69602

64 Gregis G, Sanchez JB, Bezverkhyy I, et al. Detection and quantification of lung cancer biomarkers by a micro-analytical device using a single metal oxide-based gas sensor. Sensor Actuat BChem, 2018, 255: 391-400

65 Cai QY, Zellers ET. Dual-chemiresistor GC detector employing monolayer-protected metal nanocluster interfaces. Anal Chem, 2002, 74: 3533-3539

66 Jian RS, Huang RX, Lu CJ. A micro GC detector array based on chemiresistors employing various surface functionalized monolayer-protected gold nanoparticles. Talanta, 2012, 88: 160-167

67 Bohrer FI, Covington E, Kurdak Ç, et al. Characterization of dense arrays of chemiresistor vapor sensors with submicrometer features and patterned nanoparticle interface layers. Anal Chem, 2011, 83: 3687-3695

$68 \mathrm{Mu}$ X, Covington E, Rairigh D, et al. CMOS monolithic nanoparticle-coated chemiresistor array for micro-scale gas chromatography. IEEE Sensor J, 2012, 12: 2444-2452
69 Collin WR, Serrano G, Wright LK, et al. Microfabricated gas chromatograph for rapid, trace-level determinations of gas-phase explosive marker compounds. Anal Chem, 2014, 86: 655-663

70 Tzeng T, Kuo C, Wang S, et al. A portable micro gas chromatography system for lung cancer associated volatile organic compound detection. IEEE J Solid-St Circ, 2016, 51: 259-272

71 Kim SK, Burris DR, Chang $\mathrm{H}$, et al. Microfabricated gas chromatograph for on-site determination of trichloroethylene in indoor air arising from vapor intrusion. 1. Field evaluation. Environ Sci Technol, 2012, 46: 6065-6072

72 Jian $\mathrm{M}$, Wang $\mathrm{C}$, Wang $\mathrm{Q}$, et al. Advanced carbon materials for flexible and wearable sensors. Sci China Mater, 2017, 60: 10261062

73 Lee CY, Sharma R, Radadia AD, et al. On-chip micro gas chromatograph enabled by a noncovalently functionalized single-walled carbon nanotube sensor array. Angew Chem Int Ed, 2008, 47: 5018-5021

74 Salehi-Khojin A, Lin KY, Field CR, et al. Fast carbon nanotube detectors for micro gas chromatographs. Nanoscale, 2011, 3: 30973102

75 Zhang L, Du W, Nautiyal A, et al. Recent progress on nanostructured conducting polymers and composites: synthesis, application and future aspects. Sci China Mater, 2018, 61: 303-352

76 Zheng Z, Gan L, Zhai T. Electrospun nanowire arrays for electronics and optoelectronics. Sci China Mater, 2016, 59: 200-216

77 Wanekaya AK, Uematsu M, Breimer M, et al. Multicomponent analysis of alcohol vapors using integrated gas chromatography with sensor arrays. Sensor Actuat B-Chem, 2005, 110: 41-48

78 Pirsa S, Alizadeh N. Nanoporous conducting polypyrrole gas sensor coupled to a gas chromatograph for determination of aromatic hydrocarbons using dispersive liquid-liquid microextraction method. IEEE Sensor J, 2011, 11: 3400-3405

79 Pirsa S. Design of a portable gas chromatography with a conducting polymer nanocomposite detector device and a method to analyze a gas mixture. J Sep Sci, 2017, 40: 1724-1730

80 Jiang $\mathrm{Y}$, Tang $\mathrm{N}$, Zhou C, et al. A chemiresistive sensor array from conductive polymer nanowires fabricated by nanoscale soft lithography. Nanoscale, 2018, 10: 20578-20586

81 Dziuban JA, Mróz J, Szczygielska M, et al. Portable gas chromatograph with integrated components. Sensor Actuat A-Phys, 2004, 115: 318-330

82 Qin Y, Gianchandani YB. A fully electronic microfabricated gas chromatograph with complementary capacitive detectors for indoor pollutants. Microsyst Nanoeng, 2016, 2: 15049

83 FROG-5000 http://www.defiant-tech.com

Acknowledgements The authors gratefully acknowledge the financial support from the National Natural Science Foundation of China (61674114, 91743110 and 21861132001), the National Key R\&D Program of China (2017YFF0204600), Tianjin Applied Basic Research and Advanced Technology (17JCJQJC43600), the Foundation for Talent Scientists of Nanchang Institute for Micro-technology of Tianjin University and the 111 Project (B07014 and B12015).

Author contributions $\quad \mathrm{Qu} \mathrm{H}$ wrote the paper with the support from Duan X. All authors contributed to the general discussion.

Conflict of interest The authors declare no conflict of interest. 


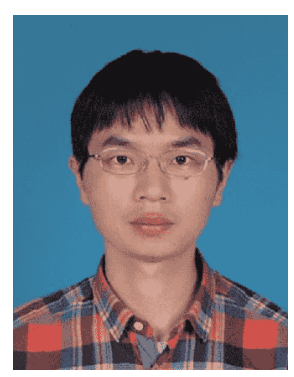

Hemi Qu is an assistant professor in Tianjin University. He received his PhD degree in chemistry at the National University of Singapore. His research interest focuses on the sensitive material for gas sensors and instrumental miniaturization.

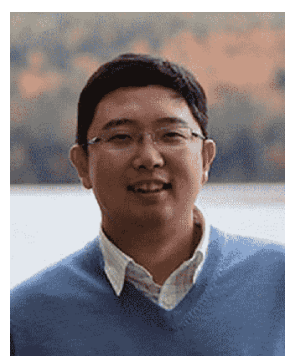

Xuexin Duan is a full professor in Tianjin University. He received his PhD degree in the Department of Molecular Nanofabrication at Universiteit Twente, Netherland. His current scientific interest focuses on nanofabrication and nanomaterials for bio-chemical sensing applications.

\section{可用于微型气相色谱仪的微型检测器研究进展}

屈贺幂 ${ }^{1,2,3}$, 段学欣 ${ }^{1,3^{*}}$

摘要 近年来, 随着安全、环境、医疗卫生等领域现场分析需求的不断增长, 分析仪器的微型化研究也日益迫切与重要. 微型气相色谱仪 具有体积小、功耗低及制作和维护成本低等诸多优点, 被认为在提供有效的现场气体分析解决方案方面具有巨大潜力. 自上世纪以来, 该 领域研究一直受到研究人员的广泛关注. 本文中, 我们将首先简要介绍微型气相色谱仪, 然后着重对可用于微型气相色谱的微型检测器的 最新研究进展进行总结. 这些进展包括常规检测器的小型化研究和新型微气相色谱检测器的研究. 